\title{
Effect of Temperature and ABA Exposure on LlaNAC Expression in Transformed Tobacco Plants
}

\section{Singh $\mathrm{S}^{*}$ and Khalid $\mathrm{H}$}

Defence Institute of Bio-Energy Research, India

*Corresponding author: Sadhana Singh, Bharathiar university, Coimbatore, India, E-

\section{Research Article}

Volume 2 Issue 3

Received Date: February 15, 2017

Published Date: February 22, 2017 mail: sadhnasingh1986@gmail.com

\section{Abstract}

NAC is one of the largest TF families in plants, with more than 100 genes per plant. NAC proteins are involved in a panorama of functions in a cell, virtually controlling the growth and development of the plants, as well as, protecting the plants against variety of stresses. We have assessed LlaNAC over-expressor (transgenic) tobacco lines (NC2, NC7b, NC10 and NC18), which were earlier displayed enhanced cold tolerance, biomass, growth and early maturity [1] in comparison to the WT. Here we report, enhanced level of relative transcript abundance in LlaNAC transformed plants to cope with heat stress together with the activation of antioxidant enzymes. The transgenic plants showed maximum of nearly two fold elevations in LlaNAC expression when exposed to temperature stress of $40^{\circ} \mathrm{C}$. Exposure to $\mathrm{ABA}(10 \mu \mathrm{M})$ caused upregulation of LlaNAC gene with an elevation of upto 1.45 fold while, higher concentration of $\mathrm{ABA}(50 \mu \mathrm{M})$ renders transgenic plants hypersentive to ABA treatments as compared to wild type plants (WT).

Keywords: ABA stress; Temperature stress; Hypersensitive; LlaNAC

\section{Introduction}

Plants are often targeted by variety of biotic and abiotic stress including salinity drought and temperature. Many genes are induced in response to these unfavorable conditions and transcription factors (TFs) are one of them [2]. These are one such gene family that upon exposure to various environmental stresses get induced. The NAC (NAM, ATAF1/2, and CUC) super family is one of the largest TF families with more than 100 copies per plant [3] participates in a number of vital processes in a plant's life cycle like development processes [4-6], stress tolerance [7], lignin synthesis [8], leaf senescence, phytohormone homeostasis $[9,10]$ etc. NAC family TFs contain a highly conserved N-terminal DNA domain and a diversified $\mathrm{C}$-terminal domain that generally regulates transcriptional activation [11,9]. In this study, LlaNAC gene was identified and cloned using a cold stress induced
cDNA library of Lepidium latifolium L. [12] in expression vector pBinAR, under the influence of promoter CaMV35S and the construct also contained $n p t I I$ gene as a selectable marker. The construct was introduced in Agrobacterium tumefaciens LBA4404 and transferred to in vitro cultures of $N$. tabacum by previous workers in the laboratory [13]. T2 seeds of four transgenic lines, viz., NC2, NC7b, NC10 and NC18 were thus available for this study. Gene expression pattern analysis demonstrated that LlaNAC transcripts was increased and followed similar pattern under heat stress and lower abscissic acid ABA $(10 \mu \mathrm{M})$ among transgenic lines.

\section{Materials and Methods}

\section{Plant Material and Growth Conditions}

Nicotiana tabacum (tobacco) plants over-expressing LlaNAC gene, and co-transformed nptII gene, (four transgenic lines-NC2, NC7b, NC10 and NC18 in 


\section{Open Access Journal of Agricultural Research}

generations T2) along with wild-type (WT) plants were maintained in containment under controlled conditions of temperature $\left(25 \pm 2^{\circ} \mathrm{C}\right)$ and light $(16 / 8 \mathrm{~h}$ photoperiod), and grown in soilrite. The plants were nourished with MS basal medium twice a week, and were watered (distilled water) on daily basis. Each individual plant was tested for its genetic stability based on herbicide tolerance assay (150 ppm paromomycin) following PCR assay with an initial denaturation at $94^{\circ} \mathrm{C}$ for $5 \mathrm{~min}$., followed by 30 cycles of $94^{\circ} \mathrm{C}$ for $30 \mathrm{~s}$ and $55^{\circ} \mathrm{C}$ for $30 \mathrm{~s}$ and $72^{\circ} \mathrm{C}$ for 1.5 min. and a final extension of $10 \mathrm{~min}$. at $72^{\circ} \mathrm{C}$. Amplicons thus obtained was run on $1 \%$ agarose gel to check the presence or absence of LlaNAC gene. For whole sets of experiments, six technical replicates were used. The native growth conditions, i.e., temperature $\left(25 \pm 2^{\circ} \mathrm{C}\right)$ and carbon dioxide (400 ppm) were considered as the control conditions.

\section{Stress Treatments and Gene Expression Analysis}

Plants of 10 week old were provided with $10 \mu \mathrm{M}$ and $50 \mu \mathrm{M}$ of ABA (Sinha et al. 2014). Regularly watered plants under optimum conditions of temperature $(25 \pm$ $\left.2^{\circ} \mathrm{C}\right)$ and light $(16 / 8 \mathrm{~h}$ photoperiod) for 1 week was considered as control. Heat stress was induced by exposing the 10 week old plants to elevated temperature of $40^{\circ} \mathrm{C}$ and light (16/8 h photoperiod) in plant growth chamber for $24 \mathrm{~h}$ following Pospisilova et al. (2011) [14], Tan et al. (2011) [15], Mackova et al. (2013) [16]. Plants under optimum conditions of temperature $\left(25 \pm 2^{\circ} \mathrm{C}\right)$ and light (16/8 $\mathrm{h}$ photoperiod) for $24 \mathrm{~h}$ were considered as control for both the extremes in temperature stress. Expressions of LlaNAC gene was analyzed by quantitative real-time polymerase chain reaction (qRT-PCR) analysis. Total RNA was isolated from young leaf tissues of control and heat exposed $\left(40^{\circ} \mathrm{C}\right) / \mathrm{ABA}$ treated $(10$ and $50 \mu \mathrm{M})$. Equal amount $(1.0 \mu \mathrm{g})$ of the RNA quantified using Qubit fluorometer (Invitrogen, USA) was used for first-strand cDNA synthesis using QuantiTect Reverse Transcription kit (Qiagen, Germany) following manufacturer's protocol. Quantitative polymerase chain reaction (qPCR) was performed using SYBR $^{\circledR}$ Green JumpStart ${ }^{\mathrm{TM}}$ Taq ReadyMix $^{\mathrm{TM}}$ (Sigma, USA) kit according to the manufacturer's instructions. Normalization reaction was carried out with an internal control actin gene using forward (5'-AGGGTTTGCTGGAGATGATG-3') and reverse (5'-ACCACGTTTG GATTGAGCTT-3') primers. The PCR amplification was carried out using the primer pair LlaNAC-TqF (5'-ACA GTG GTA AAC CTC CAA AAG G-3') and LlaNAC-TqR (5'-CGA AGA GAG TTC TTG TTG ACG A$\left.3^{\prime}\right)$ at an initial denaturation at $94^{\circ} \mathrm{C}$ for $10 \mathrm{~min}$., followed by 40 cycles of $94^{\circ} \mathrm{C}$ for $30 \mathrm{~s}$ and $55^{\circ} \mathrm{C}$ for $30 \mathrm{~s}$ and $72^{\circ} \mathrm{C}$ for $30 \mathrm{~s}$. Experiments were performed in triplicate for each set of primers. The transcript was quantified based on $\Delta \Delta \mathrm{Ct}$ method [17] and presented as fold change over control.

\section{Result and Discussion}

About $90 \%$ germination could be seen in transgenic lines grown on antibiotic medium. In the present communication, we have initiated transcriptional monitoring of stress-inducible LlaNAC gene in response to $40^{\circ} \mathrm{C}$ of heat exposure and 10 and $50 \mu \mathrm{M}$ of $\mathrm{ABA}$ application. LlaNAC expression levels were examined in leaf tissues. PCR based validations of plants were carried out using the primer pair LlaNAC-TqF (5'-ACA GTG GTA AAC CTC CAA AAG G-3') and LlaNAC-TqR (5'-CGA AGA GAG TTC TTG TTG ACG A-3') to obtain a band of size 122 bp (Figure 1).

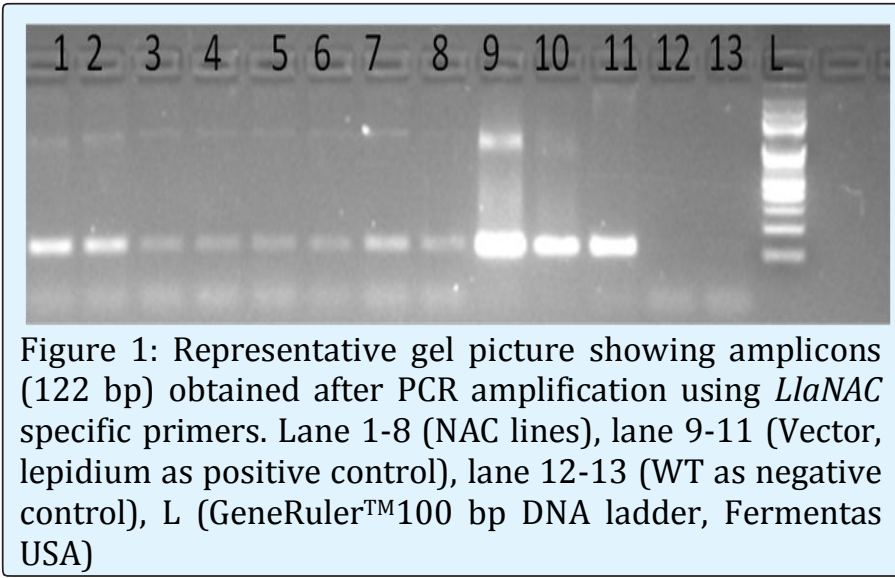

Transgenic plants found positive for the presence of LlaNAC gene along with WT were subjected to heat and ABA stress.

\section{Expression of LlaNAC Transcript under Heat Stress}

Heat stress poses a serious threat to global crop production. Plants cope with adverse temperature stress by altering molecular mechanisms and elevated antioxidants activities that decrease levels of stress inducible reactive oxygen species (ROS). Induction of NAC transcription factors in response to stresses activates expression of various stress inducible genes thereby participating in improving stress tolerance [18]. In the present study, the expression of LlaNAC gene over control was noteworthy upon heat exposure to $40^{\circ} \mathrm{C}$. Though, the amount of transcript abundance was varied among different lines. NAC Transcript expression in transgenic lines (NC10, NC7b, NC18, NC2) when exposed to 


\section{Open Access Journal of Agricultural Research}

temperature stress of $40^{\circ} \mathrm{C}$ was found significantly increased with a maximum of 1.9 fold in NC7b line, followed by an elevation of 1.4 fold in NC10 line and 1.3 fold in NC18 line over the control (Figure. 2).

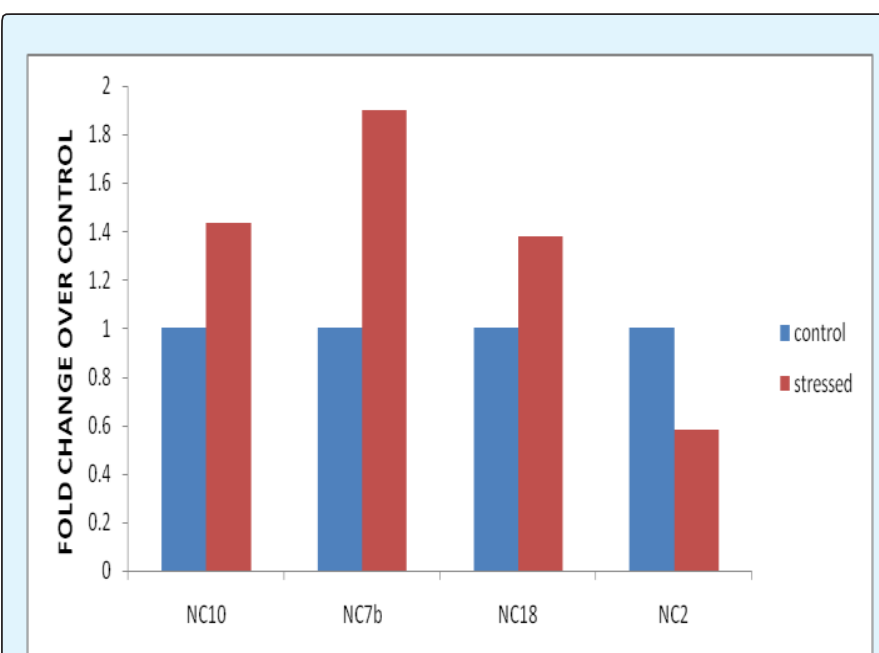

Figure 2: LlaNAC gene expression in terms of fold change over control in response to heat stress $\left(40^{\circ} \mathrm{C}\right)$ for $24 \mathrm{~h}$ against control.

Therefore, we speculated that the rapid increases in LlaNAC transcript abundance might be associated with heat tolerance in transgenic plants. However, the transcript accumulation declined in case of NC2 when compared to the control plants. Similarly, Guo et al. [19] reported up-regulation of TaNAC2L, promotes heat tolerance in transgenic arabidopsis, Han et al. [20] reported over-expression of NAC transcription factors CiNAC4 and CiNAC3 confers salinity tolerance in Arabidopsis, Hong et al. [21] reported the over-expression of ONAC022 for drought and salt tolerance in rice. Plants produce antioxidant enzymes like glutathione reductase (GR), catalase (CAT) and superoxide dismutase (SOD) to cope up with the stress induced by the increased levels of ROS. Further, we noticed elevated antioxidant enzyme activities ranging between 1.5 to 3 fold (data not shown) indicating up-regulation of LlaNAC improves heat tolerance in transgenic plants.

\section{Differential Expression of LlaNAC Transcript by ABA Treatments}

ABA is an important signaling molecule in various stresses and often considered as the secondary messenger [19]. As an important phytohormone, ABA controls various processes throughout the life cycle of plants, especially in response to external environmental stimuli $[9,18]$. Several NAC genes are involved in stress responses in an ABA-dependent manner [22], while Fang et al. [23] reported SNAC3 confers heat and drought tolerance in $\mathrm{ABA}$ independent manner. The inducibility by $\mathrm{ABA}$ was clear from the gene expression pattern at both the concentrations. Transgenic lines NC7b, NC18 and NC10 showed up-regulation of NAC gene at $10 \mu \mathrm{M}$ ABA. NAC transcript accumulation of 1.45 fold was observed in line NC7b, followed by fold of 1.37 in case of lines NC18 and NC10 over the control and again the expression of NC2 line was found repressed (Figure 3). This indicates over-expression of LlaNAC reduced ABA sensitivity at this concentration and is involved in ABA signaling. However, $50 \mu \mathrm{M}$ ABA leads to down-regulation of NAC expression in all transgenic lines (Figure 3).

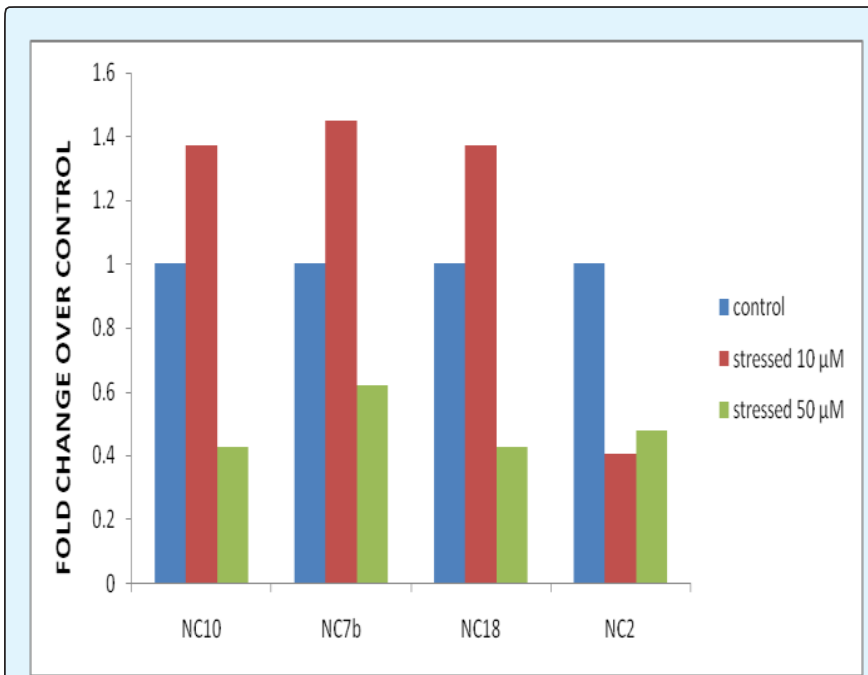

Figure 3: LlaNAC gene expression in terms of fold change over control in response to 10 and $50 \mu \mathrm{M} \mathrm{ABA}$ for one week against control.

Our results showed that plants over-expressing LlaNAC exhibited hypersensitivity to higher dose of ABA $(50 \mu \mathrm{M})$ that might be a due to lower transpiration rates and faster stomatal closure in LlaNAC over-expression plants, leading to lower water loss and enhanced tolerance. Similarly, Huang et al. [24] reported TaNAC29-over expressing arabidopsis plants exhibit ABA-hypersensitive response and its expression confers salt and drought tolerance. A relationship between ABA-hypersensitivity and abiotic stress tolerance was established. Upon treatment to $10 \mu \mathrm{M} \mathrm{ABA}$, elevated levels of GR (2.6 fold) and SOD (1.5 fold) activities were seen between transgenic and WT (data not shown). Interestingly, treatment of $50 \mu \mathrm{M}$ ABA caused no appreciable increase among transgenic lines. These results indicate LlaNAC transgenic lines exhibited ABA hypersensitivity and were positively regulated by $\mathrm{ABA}$ at higher concentrations and provide evidence that LlaNAC participates in the ABA 


\section{Open Access Journal of Agricultural Research}

signal pathway, and plays important roles in stress responses and developmental processes.

\section{Conclusion}

$N A C$ genes have widely been reported to play crucial roles in growth, development, senescence and stress responses [25] of a plant. We have here demonstrated the differential expression of the LlaNAC gene which is a transcription factor and upon binding to their respective target sites regulates the expression of various downstream genes. Over-expression of a novel NAC gene, such as LlaNAC can further offer insights into the diversity of functions that the products of these genes can play, and suitably be utilized in agricultural biotechnology.

\section{Acknowledgement}

The research work is funded by Defence Research and Development Organization (DRDO). SS and HK thanks (DRD0) for financial assistance.

\section{References}

1. Grover A, Singh S, Pandey P, Patade VY, Gupta SM, et al. (2014) Overexpression of NAC gene from Lepidium latifolium enhances biomass, shortens life cycle and induces cold stress tolerance in tobacco: potential for engineering fourth generation biofuel crops. Mol Biol Rep 41(11): 7479-7489.

2. Jiang G, Jiang X, Lü P, Liu J, Gao J, et al. (2014) The rose (Rosa hybrida) NAC Transcription Factor 3 Gene, RhNAC3, involved in ABA signaling pathway both in rose and arabidopsis. PLoS One 9(10): e109415.

3. Hu R, Qi G, Kong Y, Kong D, Gao Q et al. (2010) Comprehensive analysis of NAC domain transcription factor gene family in Populus Trichocarpa. BMC Plant Biol 10: 145.

4. Kim HS, Park BO, Yoo JH, Jung MS, Lee SM, et al. (2007a) Identification of a calmodulin-binding NAC protein (CBNAC) as a transcriptional repressor in Arabidopsis. J Biol Chem 282(50): 36292-36302.

5. Kim SG, Kim SY, Park CM (2007b) A membraneassociated NAC transcription factor regulates saltresponsive flowering via Flowering locus $\mathrm{T}$ in Arabidopsis. Planta 226(3): 647-654.

6. Kim SY, Kim SG, Kim YS, Seo PJ, Bae M, et al. (2007c) Exploring membrane-associated NAC transcription factors in Arabidopsis: implications for membrane biology in genome regulation. Nucleic Acids Res 35(1): 203-213.

7. Mao X, Chen S, Li A, Zhai C, Jing R (2014) Novel NAC Transcription Factor TaNAC67 Confers Enhanced Multi Abiotic Stress Tolerances in Arabidopsis. PLoS One 9(1): e84359.

8. Hisano H, Nandakumar R, Wang Z-Y (2009) Genetic modification of lignin biosynthesis for improved biofuel production. In Vitro Cell Dev Biol plant 45(3): 306-313.

9. Puranik S, Sahu PP, Srivastava PS, Prasad M (2012) NAC proteins: regulation 13 and role in stress tolerance. Trends Plant Sci 17(6): 369-381.

10. Nakashima K, Takasaki H, Mizoi J, Shinozaki K, Yamaguchi-Shinozaki K (2012) NAC transcription factors in plant abiotic stress responses. Biochimi Biophys Acta 1819(2): 97-103.

11. Ernst HA, Olsen AN, Larsen S, Lo Leggio L (2004) Structure of the conserved domain of ANAC, a member of the NAC family of transcription factors. EMBO Rep 5(3): 297-303.

12. Aslam M, Sinha VB (2010) Isolation of cold stress responsive genes from Lepidium latifolium by suppressive subtraction hybridization. Acta Physiol Plant 32(1): 205-210.

13. Aslam M, Grover A, Sinha VB, Fakher B, Pande V, et al. (2012) Isolation and characterization of cold responsive NAC gene from Lepidium latifolium. Mol Biol Rep 39(10): 9629-9638.

14. Pospisilova J, Haisel D, Vankova R (2011) Responses of transgenic tobacco plants with increased proline content to drought and/or heat stress. Am J Plant Sci 2(3): 318-324.

15. Tan W, Meng Qw, Brestic M, Olsovska K, Yang X (2011) Photosynthesis is improved by exogenous calcium in heat-stressed tobacco plants. J Plant Physiol 168(17): 2063-2071.

16. Macková $H$, Hronková $M$, Dobrá J, Turečková V, Novák 0 , et al. (2013) Enhanced drought and heat stress tolerance of tobacco plants with ectopically enhanced cytokinin oxidase/dehydrogenase gene expression. J Exp Bot 64(10): 2805-2815. 


\section{Open Access Journal of Agricultural Research}

17. Livak KJ, Schmittgen TD (2001) Analysis of relative gene expression data using a real-time quantitative

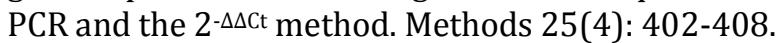

18. Basu S, Roychoudhaury A (2014) Expression profiling of abiotic stress-inducible genes in response to multiple stresses in rice (oryza sativa L.) varieties with contrasting level of stress tolerance. BioMed Research International 12 Article ID 706890.

19. Guo W, Zhang G, Zhang N, Xin M, Peng H, et al. (2015) The wheat $\mathrm{NAC}$ transcription factor $\mathrm{TaNAC}_{2} \mathrm{~L}$ is regulated at the transcriptional and post-translational levels and promotes heat stress tolerance in transgenic arabidopsis. PLoS One 10(8): e0135667.

20. Han X, Feng Z, Xing D, Yang Qi, Wang R, et al. (2015) Two NAC transcription factors from caragana intermedia altered salt tolerance of the transgenic arabidosis. BMC Plant Biology 15: 208.

21. Hong Y, Zhang H, Huang L, Li D, Song F (2016) Overexpression of a stress-responsive NAC transcription factor gene ONAC022 improves drought and salt tolerance in rice. Front Plant Sci 7: 4.
22. Fujita Y, Fujita M, Shinozaki K, Yamaguchi-Shinozaki $\mathrm{K}$ (2011) ABA-mediated transcriptional regulation in response to osmotic stress in plants. J Plant Res 124(4): 509-25.

23. Fang Y, Liao K, Du H, Xu Y, Song H, et al. (2015) A stress-repsonsive NAC transcription factor SNAC3 confers heat and drought tolerance through modulation of reactive oxygen species in rice. J Exp Bot 66(21): 6803-6817.

24. Huang Q Wang Y, Li B, Chang J, Chen M, et al. (2015) TaNAC29, a NAC transcription factor from wheat, enhances salt and drought tolerance in transgenic arabidopsis. BMC Plant Biology 15: 268.

25. Sinha VB, Grover A, Aslam M, Ahmed Z, Pande V (2014) Isolation and characterization of Ras related GTP binding protein (Ran) from Lepidium latifolium $\mathrm{L}$. reveals its potential role in regulating abiotic stress tolerance. Acta Physiol Plant 36(9): 2353-2360. 